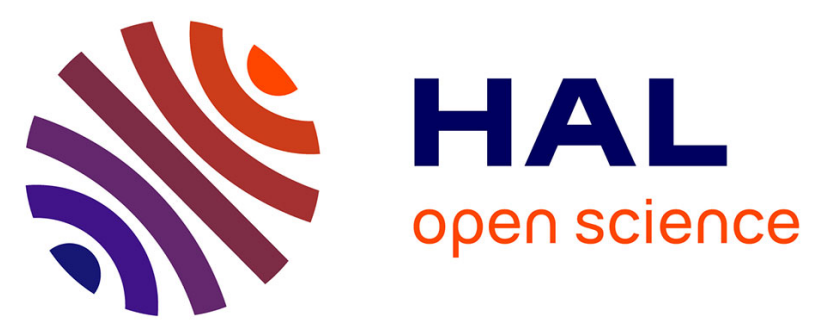

\title{
Description des régulations opérées par les soignants face aux facteurs organisationnels: un enjeu de santé au travail et de performance des services de soins
}

Elsa Laneyrie, Sandrine Caroly, Alexis Descatha

\section{- To cite this version:}

Elsa Laneyrie, Sandrine Caroly, Alexis Descatha. Description des régulations opérées par les soignants face aux facteurs organisationnels: un enjeu de santé au travail et de performance des services de soins. Archives des Maladies Professionnelles et de L'Environnement, 2018, 80 (1), pp.8-15. 10.1016/j.admp.2018.10.003 . hal-02005262

\section{HAL Id: hal-02005262 \\ https://hal.science/hal-02005262}

Submitted on 21 Oct 2021

HAL is a multi-disciplinary open access archive for the deposit and dissemination of scientific research documents, whether they are published or not. The documents may come from teaching and research institutions in France or abroad, or from public or private research centers.
L'archive ouverte pluridisciplinaire HAL, est destinée au dépôt et à la diffusion de documents scientifiques de niveau recherche, publiés ou non, émanant des établissements d'enseignement et de recherche français ou étrangers, des laboratoires publics ou privés.

\section{(ㄷ)(1) $\$$}

Distributed under a Creative Commons Attribution - NonCommerciall 4.0 International 


\section{Description des régulations opérées par les soignants face aux facteurs organisationnels : un enjeu de santé au travail et de performance des services de soins}

\section{Description of the regulations made by the caregivers in the face of organizational factors: a challenge of occupational health and performance of care services}

\section{Summary :}

Objectives of the study. Show how the organizational context influences the type of regulation of the activity implemented by the nurses during their activity in order to maintain the performance in service of care while maintaining its own health.

Method. An ergonomic-type activity analysis of six nurses was conducted in three geriatric care units. Following these open observations, a thematic analysis was carried out.

Results. The analysis of our data consisted in identifying three types of regulation used by the nurses: time management, space and material management and finally the management of the workload by the collective work contained in the organizational context care services.

Conclusion. This article enriches the many research on the work of caregivers by linking the organizational context with the type of regulation of the activity used by caregivers to maintain the productivity of the care service and preserve their health.

\section{Key words: Regulation, Nurses, organizational context}

\section{Résumé :}

Objectifs de l'étude. Montrer comment le contexte organisationnel influence le type de régulations de l'activité mise en œuvre par les soignants au cours de leur activité afin de maintenir la performance en service de soins tout en maintenant sa propre santé.

Méthode. Une analyse de l'activité de type ergonomique auprès de six soignants a été effectuée au sein de trois services de soins de gériatrie. Suite à ces observations ouvertes, une analyse thématique a été réalisée.

Résultats. L'analyse de nos données a consisté à identifier trois types de régulation utilisé par les soignants soit la gestion du temps, la gestion de l'espace et du matériel et enfin la gestion de la charge de travail par le travail collectif compte-tenu du contexte organisationnel des services de soins.

Conclusion.

Cet article permet d'enrichir les nombreuses recherches sur le travail des soignants en mettant en relation le contexte organisationnel avec le type de régulations de l'activité utilisé par les soignants pour maintenir la productivité du service de soins et préserver leur santé.

\section{Mots clés : Régulation, Soignant, contexte organisationnel}




\section{Introduction}

L'organisation hospitalière est confrontée en ce début du XXIème siècle à des contraintes s'apparentant à celles que rencontrent les salariés dans les entreprises privées [1]. Plusieurs évolutions économiques et sociales touchent le milieu hospitalier. Ainsi, l'hôpital doit aujourd'hui répondre à des objectifs de rentabilité [la réforme de la Tarification à l'Acte en est un exemple ainsi que le plan hôpital 2007)] $]^{1}$ modifiant fortement le système de valeurs, les pratiques professionnelles des soignants [2] ainsi que l'organisation du travail au sein des services de soins.

\section{Etre soignants dans le service public aujourd'hui}

Le travail des soignants est décrit comme contraignant si l'on se réfère aux critères de pénibilité définis par le code du travail (article 4121-3-1). Trois critères de pénibilité caractérisent le milieu soignant: des contraintes physiques marquées (manutentions, postures pénibles...) ; un environnement physique agressif (agents chimiques dangereux, bruits...) et enfin, un rythme de travail atypique (travail de nuit, travail répétitif, travail en équipes successives alternantes...). Ainsi, le lien entre contraintes organisationnelles en milieu de soins et troubles musculo-squelettiques (TMS) est avéré scientifiquement [3 -4-5-6]. Plusieurs études montrent notamment qu'une organisation du travail mal adaptée à l'activité des soignants est source d'interruptions de tâches [7], de confusions de rôle entre les professionnels, et d'augmentation de la charge de travail [8-9-10]. A ce titre, l'intensification du travail, le travail répétitif, ou encore la faible reconnaissance du travail réalisé par les managers ont un impact sur le bien être des soignants [11].

Ce mal-être généralisé de la population est d'autant plus prégnant à prendre en considération que nous faisons face aujourd'hui à une pénurie de personnel et à une population active vieillissante [12]. Ainsi, les établissements ont à faire face à un taux d'absentéisme significatif [13-14] et à des inaptitudes partielles en augmentation constante [2] mettant ainsi en difficulté la gestion de l'organisation du travail, voire la qualité du service rendu auprès des patients.

Face à ce constat et aux différents types de variabilités inhérentes à l'activité de travail (organisationnelles, techniques et humaines), les soignants ne restent pas passifs face aux tâches qui leurs sont demandées. Ils vont rechercher un équilibre entre la performance qui leur est demandée et la préservation de leur propre santé dans le but de répondre aux obligations

\footnotetext{
${ }^{1}$ http://www.vie-publique.fr/politiques-publiques/politique-hospitaliere/gouvernance-hospitaliere/
} 
de production, de réduire les risques d'erreurs, d'incidents, d'accidents ou de débordements [15]. Cette recherche d'équilibre se nomme la régulation [16]. Les régulations effectuées par les opérateurs prennent différentes formes telles que des adaptations, des détournements, des transformations de procédures [17]. A ce titre, les régulations permettent aux individus de modifier certains moyens de travail, d'augmenter des ressources personnelles, d'élargir leur réseau professionnel et le travail en équipe par exemple.

L'objectif de ce papier est alors de décrire l'activité d'organisation par les soignants de leur propre travail face aux nombreuses contraintes organisationnelles présentes en milieu de soins. L'hypothèse sous-jacente est que l'identification des contraintes organisationnelles en service de soins et les régulations mises en œuvre par les soignants pour y pallier, permettraient de concevoir une prévention plus adaptée dans les services de soins et plus efficace, permettant ainsi de réduire les risques professionnels auxquels cette population est confrontée.

\section{Méthode afin de décrire l'activité d'organisation des soignants de leur propre travail}

L'analyse de l'activité de type ergonomique effectuée a consisté à faire des observations de terrain. Nous avons noté dans le moindre détail tous les faits, les gestes et échanges des soignants entre eux. Ces observations étaient de type papier crayon (6 jours soit 48 heures par service) au sein de trois services de soins de gériatrie ${ }^{2}$ auprès de 6 soignants différents durant le service du matin $(6 \mathrm{H} 30-14 \mathrm{H} 30)$ car cette période est réputée comme la plus dense de la journée par les professionnels [18]. Ces observations ont été complétées par des entretiens informels avec les soignants. Nous avons choisi des profils différents en termes de profession (IDE, AS), d'ancienneté dans la profession, d'ancienneté dans le service et d'âge afin d'avoir une représentativité dans nos données.

Tableau 1. Caractéristiques de l'échantillon

L'analyse de type qualitative des données recueillies s'est effectuée en trois temps :

- Premièrement, nous avons caractérisé les contextes organisationnels des services de soins observés en fonction de 5 facteurs catégorisés dans la littérature comme pertinent [3] (cf. tableau 2) :

\footnotetext{
${ }^{2}$ Plusieurs spécialistes s'accordent à dire que c'est au sein de ces services que les conditions de travail sont les plus difficiles d'un point de vue physique (manutention) et psychique (patients en fin de vie, troubles cognitifs, dépendances...) [3].
} 
○ l'organisation des services de soins

- la gestion des patients (le nombre et le type de patients)

- la présence de lève-malade dans le service de soins (proportion de lève malade en fonction du nombre de patients),

- les projets de service en cours (projets en cours et temps de communication avec l'équipe),

- la politique en matière de ressources humaines de l'établissement (glissement de tâche et travail en binôme autorisé, gestion de l'absentéisme...).

Tableau 2. Contextes organisationnels des services de soins observés

- Secondement, nous avons identifié trois types de régulations utilisés par les soignants au cours de leur activité de travail :

○ la gestion du temps, comprenant toutes les stratégies des soignants afin de gagner du temps.

- la gestion de l'espace et du matériel, comprenant toutes les stratégies des soignants afin d'utiliser le matériel à disposition dans le service de soins.

- la gestion de la charge de travail par le travail collectif, comprenant toutes les stratégies des soignants afin de travailler en binôme. Afin de les déterminer, nous avons analysé le type de communication utilisée par le soignant observé (AS ou IDE) durant son service. Nous avons catégorisé ces échanges selon 4 catégories :

- L'aide : le soignant observé demande de l'aide durant son activité.

- Espace et matériel : le soignant observé demande des informations sur du matériel.

- Organisation des soins entre différents types de professionnels (AS et IDE) : le soignant observé organise sa charge de travail avec un collègue pratiquant un autre métier.

- Coordination des soins entre mêmes professionnels (AS/AS et IDE/IDE) : le soignant observé organise sa charge de travail avec un collègue pratiquant le même métier.

- Enfin, nous avons analysé comment le contexte organisationnel des services de soins (décrit dans le tableau 2) pouvait avoir un impact sur le type de régulation utilisé par les soignants au cours de leur activité de travail. 


\section{Résultats : Description des régulations de l'activité utilisées par les soignants}

Cette partie vise à rapprocher le type de régulations mises en œuvre par les soignants observés au cours de leur activité en fonction du contexte organisationnel du service de soins (décrits dans le tableau 2). Etant donné les disparités contextuelles entre les services, nous ne comparerons pas les services entre eux. Notre objectif est de montrer, comme un panel, comment le contexte d'un service peut faire émerger des régulations de l'activité chez les soignants afin de gérer les contraintes de temps et de matériel ainsi que la charge de travail quotidienne. Pour faire cela, nous centrerons principalement l'analyse de nos données sur le travail des IDE dans les services 1 et 3 et sur celui des AS dans le service 2 car ce sont auprès de ces acteurs que les régulations sont les plus importantes. Rappelons que les régulations utilisées par les soignants visent à répondre aux fortes exigences organisationnelles et aux diverses pressions temporelles et ce afin de maintenir la productivité du service (continuité des soins, qualité des soins administrés) et préserver leur propre santé.

\section{Des régulations de l'activité relatives à la gestion du temps}

Avant de détailler pour chacun des services observés, les régulations utilisées par les soignants durant leur activité de travail relative à la gestion du temps, nous souhaitons présenter plusieurs «techniques communes » observées, quant aux toilettes des patients, utilisées par les soignants : les patients sont lavés tous les deux jours; seules les parties intimes du corps des patients sont lavées; les soignants ne laissent pas les patients se laver seuls; les toilettes sont principalement alitées; les soins de bouche sont reportés. Ces différentes techniques au bénéfice de la performance du service de soins ont un impact considérable sur la qualité des soins réalisés.

Plus spécifiquement, concernant le service de soins 1, contenu de l'absentéisme récurrent et de la politique en matière de ressources humaines visant à inciter les soignants à travailler au maximum seuls, les soignants travaillent sans arrêt dans l'anticipation des soins. En effet, l'analyse des écarts entre la fiche de poste des infirmiers (IDE) et l'activité observée montre qu'ils commencent 30 minutes plus tôt leur travail par rapport à ce qui leur est demandé afin de pouvoir terminer à l'heure leur service. Nous observons aussi que le temps de transmission entre l'équipe de nuit et du matin dure 15 minutes de moins que ce qui est prescrit (30 minutes). Cette réduction permet à la fois aux collègues de nuit de partir plus tôt et à l'équipe du matin de «gagner du temps » sur la préparation des médicaments. Les deux IDE du matin 
préparent alors ensemble les médicaments nécessaires pour leur première tournée. De plus, toujours dans un souci d'anticipation du temps, les IDE essaient de commencer leur tournée de distribution de médicaments avant que les aides-soignants (AS) distribuent les petits déjeuners. Ceci leur permet de laisser les médicaments sur la table de nuit des patients afin que les AS les donnent pendant le petit déjeuner ou le repas. Les IDE essaient de compenser la pression temporelle ressentie en commençant leur tournée plus tôt que celle des AS le matin. Dans ce service, le travail collectif s'opère principalement entre les IDE et les AS, les ASH sont peu présentes.

Au sein du service de soins 2, comme il est autorisé informellement aux ASH d'effectuer des tâches d'AS (qui ne leurs sont donc pas attribuées légalement : glissement de tâches), les AS arrivent à répondre aux exigences attendues. A ce titre, les ASH préparent les petits déjeuners, ce qui permet aux AS de commencer les toilettes plus tôt. Les ASH aident aussi à lever les patients les plus autonomes afin qu'ils puissent prendre leur petit déjeuner seuls. Les AS eux, s'occupent des patients les plus «lourds». Ceci permet à l'équipe de gagner du temps, les patients étant levés, réveillés, l'ensemble des professionnels peut ainsi commencer son tour de soins. Dans ce service, le travail collectif (soit travailler à plusieurs sur un objet commun) s'opère principalement entre les AS et les ASH, c'est pourquoi nous ne parlerons pas de l'activité des IDE.

Au sein du service de soins 3, l'absentéisme fréquent et le manque de remplacement au sein du service de soins amènent les IDE à renforcer leurs collaborations. Par exemple, bien que cela ne soit pas permis par le protocole de soins, les IDE de nuit préparent les médicaments (transfusions, injections...) nécessaires lors de la distribution du matin pour les patients. Cet écart avec le prescrit du protocole permet aux IDE du matin de gagner du temps et commencer ainsi directement leur tournée de soins. Ceci témoigne de liens de confiance au sein de l'équipe et d'entraide entre les professionnels. Enfin, nous avons observé que la transmission du matin est écourtée de 10 minutes par rapport aux 30 minutes normalement exigées.

L'analyse de contexte au regard des régulations utilisées par les soignants montre plusieurs indicateurs favorables à la performance des services de soins : comme le fait d'autoriser certains soignants à collaborer entre professionnels pour la réalisation des soins ou encore le fait de donner la permission aux ASH d'aider les soignants. Cependant, le glissement de tâches interroge la qualité des soins dispensés (les ASH n'ont pas eu de formation sur la 
manière de se positionner vis-à-vis des patients, de les lever ou encore les nourrir), la responsabilité professionnelle, voire pénale en cas d'accident du travail, ou d'erreur d'administration de médicaments est posée.

A l'inverse, les indicateurs défavorables à la performance des services de soins sont relatifs à l'absentéisme et au manque de remplacement des soignants absents. Si ces problématiques sont trop récurrentes elles vont avoir un impact sur la santé des soignants. A ce titre, plusieurs formes de régulations liées à la gestion du temps durant l'activité des soignants sont observées : relatives au temps de travail (commencer plus tôt sa journée de travail, diminuer le temps de transmission entre l'équipe de nuit et du matin, débuter la tournée des médicaments avant celle des AS), à la préparation des médicaments (préparation conjointe des IDE avant de commencer leurs tournées, préparation par l'équipe de nuit), à l'implication des ASH (glissement de tâche des ASH) ou encore à la manière de réaliser les toilettes. Ces différentes formes de compensation questionnent la performance du service proposé aux patients car elles ne permettent pas toujours la réalisation d'un travail pensé, de qualité mais surtout sûr du point de vue de leur santé.

\section{Des régulations de l'activité relatives à la gestion de l'espace et du matériel}

Avant de détailler pour chacun des services observés, les régulations, utilisées par les soignants durant leur activité de travail relatives à l'espace et au matériel, nous avons identifié que les soignants des trois services de soins observés utilisent «la technique du drap » afin de changer les draps souillés des patients. Cette technique permet d'économiser les efforts des soignants en mobilisant lorsque cela est possible les patients. Cependant, au sein du terrain 1, les patients sont habillés (tandis que dans les services 2 et 3 les patients sont en chemise) ce qui entraine une charge de travail supplémentaire pour les soignants.

Plus spécifiquement au sein du service 1, un lève malade pour 35 patients est recensé alors que les patients sont très dépendants (seuls 4 patients sont en capacité de se lever seuls de leur lit durant nos observations). De plus, ce lève-malade ne semble pas toujours adapté aux pathologies des patients, plusieurs d'entre eux se sont plaints de douleurs liées aux sangles de l'appareil. Nous avons pu aussi observer que cet appareil, fonctionnant à l'aide de batteries, a pendant son utilisation été en panne laissant le patient quelques minutes en l'air, au-dessus de son lit. Le manque de lève malade dans le service conduit les soignants à réguler en s'organisant entre eux dans le déplacement de ce matériel, d'une aile à l'autre du bâtiment. Il convient ainsi d'organiser la charge en soins du service non plus uniquement entre collègues d'une même aile mais aussi en fonction des patients de la seconde aile. L'organisation choisie 
en amont, dès que cela est possible, est de rassembler les patients les plus dépendants non pas dans la même aile mais le plus près possible des bureaux infirmiers : limitrophes aux deux ailes. Ceci permet alors de ne pas avoir un secteur réputé comme plus lourd et d'alléger la charge en soins des soignants. Concrètement, au quotidien, les soignants utilisent, aile après aile, le lève-malade. Chaque jour, l'ordre des ailes utilisatrices s'inverse. Cependant, lorsque les patients ont des rendez-vous, des analyses, l'organisation des soignants est modifiée et il convient alors de préparer les patients le plus rapidement possible et ce quelle que soit l'aile dans laquelle ils sont situés. L'ensemble de ces contraintes pousse les soignants à devoir manipuler sans lève-malade les patients et ce majoritairement sans l'aide de collègues.

Au sein du service 2, bien que le type de patient atteint de la maladie d'Alzheimer soit fréquent, il n'y a pas de lits adaptés ${ }^{3}$. Afin de limiter le risque de chute des patients, les soignants régulent et mettent les matelas à terre. Cependant, cette stratégie n'est pas satisfaisante pour les soignants, car ceci modifie considérablement le type de prise en charge du patient et la façon d'exercer son métier en termes d'actes techniques, de gestuelle (contraintes biomécaniques plus importantes afin de réaliser les soins) mais aussi de valeurs professionnelles (respect de la dignité du patient : acte pouvant être considéré comme de la maltraitance).

Enfin, afin de faire face à un manque récurrent d'effectifs, les équipes ont créé un outil compromettant le secret professionnel : il se présente sous la forme d'un tableau disposé sur le chariot de linge des AS dans le couloir, et donne les caractéristiques de chaque patient : taille des protections, préférences alimentaires... Cet outil permet aux personnels en formation, ou remplaçants d'être autonomes sur le poste mais aussi aux titulaires d'avoir le matériel nécessaire sur le chariot correspondant aux besoins des patients. Rappelons que les soignants doivent calculer au plus prés le nombre de draps, protections... nécessaire pour un patient dans une chambre. Par mesure d'hygiène, ils ne peuvent pas se resservir du matériel non utilisé pour d'autres chambres. Cet outil a donc des fonctions logistiques et économiques.

Au sein du service 3, le lève-malade n'est pas adapté à la corpulence des patients, et ne rentre pas dans toutes les chambres. Ainsi les soignants ne l'utilisent pas et lèvent les patients manuellement principalement. Les manutentions se font souvent seuls car le service est confronté à un absentéisme important augmentant la charge en soins des soignants. Ceci est

\footnotetext{
${ }^{3}$ Ces lits sont surbaissés pour limiter les chutes, sans barrière afin que le patient ne les escalade pas. Ils sont de plus adaptés aux personnels soignants qui peuvent le remonter à leur convenance afin de réaliser les soins.
} 
renforcé par le fait que les soignants sont sectorisés. Demander de l'aide à un collègue nécessite d'aller le chercher dans un autre secteur, l'interrompre dans son activité, attendre que ce dernier ait terminé. Ceci est donc couteux.

L'analyse de contexte au regard des régulations utilisées par les soignants montre plusieurs indicateurs favorables à la performance des services de soins : comme le fait de mettre à disposition des soignants du matériel en nombre, adapté et fonctionnant, et d'organiser les services de soins par couloirs et non pas par ailes. En mode dégradé, plusieurs régulations sont identifiées : distribuer les patients dans les services (égaliser la charge en soins entre les ailes, rapprocher les patients les plus lourds du bureau infirmer), manipuler les patients (seul ou en collectif, avec ou sans un lève malade, ou à terre si absence de lit efficient compte tenu de la pathologie du patient) ou encore celles relevant de la création d'un outil logistique d'aide.

\section{Des régulations collectives relatives à la manière de se distribuer la charge de travail}

Nous nous sommes intéressés particulièrement à la manière dont les soignants communiquent entre eux afin de se distribuer la charge de travail tout au long de leur prise de fonction. Afin de présenter les données recueillies lors de nos observations nous les avons classées sous la forme d'un tableau (cf. tableau 3), en fonction de 4 types d'échange : relatifs à l'aide, aux matériels, à l'organisation et la coordination des soins

Tableau 3. Catégorisation des échanges des soignants observés afin de se répartir la charge de travail

Les chiffres du tableau correspondent aux proportions, soit le nombre de fois que le professionnel observé a utilisé un type d'échange en particulier. Une première lecture du tableau montre que les soignants (IDE et AS) observés utilisent majoritairement le registre de la coordination des soins dans leur activité.

Au sein du service 1, le fait que le service soit organisé en ailes et que la politique du service invite les soignants à effectuer seuls les toilettes des patients, influe sur le type d'échanges entre les professionnels. En effet, l'analyse des échanges montre que les AS et les IDE utilisent principalement le même registre de communication soit la coordination des soins entre pairs. Seuls durant leur activité, les soignants (tant AS qu'IDE) ont besoin de faire des points réguliers entre eux afin de se répartir la charge en soins. Nous observons cependant 
une différence notable entre les IDE et les AS concernant les échanges autour du matériel. Ceci peut s'expliquer par le fait que plusieurs IDE ont été nouvellement affectés dans le service et ont donc des questions à poser.

Au sein du service 2, la possibilité donnée aux AS de travailler en binôme toute la matinée, aux ASH d'aider les AS et ce au sein d'un service de soins organisé en couloir (avec des chambres de chaque côté permettant plus facilement l'échange d'informations) influe le type d'échanges des soignants. En effet, les AS utilisent principalement le registre de l'organisation des soins entre différents professionnels (AS, IDE) tandis que les IDE utilisent principalement le registre de la coordination (entre IDE). Ces différences s'expliquent par le fait que les AS ont moins besoin de se coordonner entre eux afin de se répartir la charge en soins puisqu'ils travaillent déjà en binôme (entre AS). Ils ont cependant besoin de se coordonner avec les IDE. Les IDE, moins nombreux, travaillent plus souvent seuls et ont besoin de se coordonner entre eux afin de se répartir la charge en soins. Ainsi, les IDE utilisent plus souvent le registre de l'aide car travaillant plus souvent seuls, ils demandent de l'aide aux collègues. Enfin, les IDE ont plus souvent des échanges portant sur les matériels que les AS. Ceci peut s'expliquer par le fait que les AS travaillant souvent en binôme n'utilisent pas systématiquement le lèvemalade, l'IDE étant seul, est davantage obligé à s'en servir.

Au sein du service 3, le travail collectif n'est pas encouragé par la politique institutionnelle. Ainsi, les AS et les IDE utilisent majoritairement le même registre de la coordination des soins afin de s'organiser collectivement pour se répartir la charge en soins. Ainsi, les soignants sont clivés dans leurs tâches respectives et communiquent peu avec des collègues d'autres métiers. Nous observons de plus que les IDE demandent plus fréquemment de l'aide aux collègues et ont besoin de plus s'organiser entre eux. Ceci peut s'expliquer par le fait qu'il y ait des nouveaux infirmiers dans le service.

Pour conclure, les facteurs organisationnels ont un impact sur l'organisation de la charge en soins des soignants, ce qui influence alors le destinataire (entre pairs, entre différents corps de métier), le nombre et type d'échanges (demande d'aide, recherche de matériel, organisation de la charge de travail entre pairs ou entre IDE/AS) durant leur service.

\section{Discussion}

L'objectif sous-jacent de cet article est de contribuer aux recherches postulant que le fait de 
mieux comprendre l'interaction entre le contexte organisationnel au sein d'un service et les régulations utilisées par les opérateurs, permet d'adapter la démarche préventive mise en œuvre de façon plus efficiente. Pour faire cela, il est nécessaire à la fois de décrire le contexte du service observé (empreint de facteurs organisationnels) puis les régulations y étant associées.

\section{Facteurs organisationnels à repérer en service de soins}

Selon Berthelette, Bilodeau et Leduc [19] utilisant la définition de Scheirer (1987) le contexte est défini comme «les caractéristiques sociales, politiques, organisationnelles et culturelles des milieux de travail dans lesquels les interventions ont été implantées »(p.174). Plus largement, le contexte d'un établissement comprend des facteurs internes et externes à 1'entreprise. Le contexte interne de l'entreprise est constitué des caractéristiques de l'établissement : soit la taille de l'entreprise, la stabilité ou l'instabilité d'emploi, le climat social, le type de management, le niveau de qualification de la main-d'œuvre, de l'organisation et des activités en prévention de l'établissement ..., tandis que le contexte

externe est composé du contexte économique général, du contexte réglementaire et législatif [20-21]. Dans le cadre de cette recherche, nous ne nous attardons pas sur le contexte externe puisqu'il est similaire aux trois terrains observés. Cependant, 5 facteurs organisationnels ont été retenus afin de caractériser le contexte interne d'un service de soins relatifs à : l'organisation du service de soins, l'autonomie des patients, la proportion de lève malade disponible, aux projets de service, et la politique des ressources humaines. La prise en compte de ces facteurs est importante car une organisation «qui ne faciliterait pas les processus de régulations dans le travail [22], voire qui les nierait, provoquerait une dégradation du travail, dans le sens où elle ne permettrait pas les débats nécessaires à sa remise en cause permanente » $(\mathrm{p} 222)[23]$.

\section{Les régulations mises en œuvre par les soignants}

Trois types de régulation ont été identifiés au regard du contexte organisationnel des services de soins observés : la gestion du temps, la gestion de l'espace et du matériel et enfin la gestion de la charge de travail par le travail collectif. Ces régulations sont mises en œuvre par les soignants dans le but de maintenir la performance du service de soins et protéger sa propre santé. Cependant, elles ne sont pas toujours efficaces. Si les régulations ne sont pas identifiées par les managers, ni prises en compte par l'organisation du travail, ou encore si l'organisation du travail est trop prescriptive, cette dernière va empêcher les régulations. Clot parle d'activité empêchée [24]. Cet empêchement va avoir un coût en termes de santé pour les opérateurs 
(risque de troubles musculosquelettiques (via le transfert seul des patients, le port de charge par exemple..), de risques psychosociaux (perte de sens dans le travail réalisé, isolement dans son activité, stress permanent afin d'atteindre les objectifs fixés...) [25] et de perte de performance pour l'entreprise (la qualité et sécurité des soins administrés peuvent être mis en péril notamment via les glissements de tâches) [26].

En revanche, lorsque l'organisation du travail laisse suffisamment de marges de manœuvre aux soignants, elle leur permet de réguler leur activité [27] face aux différents types de variabilités, ce qui a un effet protecteur sur leur santé. Les soignants mobilisent leur pouvoir d'agir [28] dans leur situation de travail. Cette mobilisation va favoriser le travail collectif des soignants [29] autour d'un projet commun, ce qui favorise la productivité du service de soins (discussion autour des règles de métier, amélioration des procédures...). Cependant, ce travail collectif en service de soins doit être soutenu institutionnellement et accompagné par des espaces de parole, la possibilité de travailler en binôme, l'accès à des formations... Caroly et Clot [30] montrent qu'un travail collectif efficient au sein d'une équipe prévient les troubles psychosociaux et biomécaniques.

\section{Conclusion}

Cet article permet d'enrichir les nombreuses recherches sur le travail des soignants en mettant en relation le contexte organisationnel avec le type de régulations de l'activité utilisé par les soignants pour maintenir la productivité du service de soins et préserver leur santé. Rappelons qu'un soignant épanoui au travail est un professionnel en bonne santé mais aussi productif. L'un ne va pas sans l'autre. C'est notamment par la productivité que le soignant développe sa pratique mais aussi l'organisation dans laquelle il interagit. Il convient ainsi de trouver le bon équilibre entre ces deux dimensions. C'est pourquoi l'approche pluridisciplinaire de l'analyse des situations de travail est déterminante car elle permet d'identifier l'ensemble des facteurs pertinents et ayant un impact sur l'activité de travail des soignants.

\section{Déclaration de liens d'intérêt}

A. Descatha perçoit des droits d'auteurs d'Elsevier Masson pour son activité au sein de la rédaction des Archives des maladies professionnelles et de l'environnement. Les autres auteurs déclarent ne pas avoir de liens d'intérêts.

\section{Remerciements :}

Nous tenons à remercier la CNRACL ainsi que tous les professionnels de santé ayant participé à cette étude. 


\section{Bibliographie :}

[20] Baril-Gingras, G., Bellemare, M., \& Brun, J.P. (2004). Intervention externe en santé et en sécurité du travail. Un modèle pour comprendre la production de transformations à partir de l'analyse d'interventions d'associations sectorielles paritaires (Rapport $\left.\mathrm{n}^{\circ} 367\right)$. Montréal: IRSST.

[19] Berthelette, D., Bilodeau, H., \& Leduc, N. (2008). Pour améliorer la recherche évaluative en santé au travail. Santé Publique, 20(3), 171-179.

[14] Brami, L., Damart, S., \& Kletz, F. (2013). Santé au travail et travail en santé. La performance des établissements de santé face à l'absentéisme et au bien-être des personnels soignants. Management et avenir. 61(3), 168-189.

[17] Caroly, S. (2002). Gérer les règles et le client : implication des cadres dans les modalités d'ajustement des règles pour une organisation efficiente. Communication présentée lors du 37ème congrès de la SELF, Aix-en-Provence.

[30] Caroly, S., \& Clot, Y. (2004). Du travail collectif au collectif de travail. Des conditions de développement des stratégies d'expérience. Comparaison de deux bureaux. Formation et Emploi, 1(88), 43-55.

[29] Caroly, S. (2010). L'activité collective et la réélaboration des règles : des enjeux pour la santé au travail (Habilitation à diriger des recherches, Université Victor Segalen Bordeaux II, Bordeaux, France).

[6] Caroly, S., Moisan, S., Juret, I. \& Roquelaure, Y. (2011). Les artefacts et instruments médicaux. Dans J. Dinet, et C. Batien (Edit.). Ergonomie des objets et environnements physiques et numériques. France : Lavoisier.

[12] Chiron, E. (2008). Les TMS et le maintien en emploi des salariés de 50 ans et plus: un défi pour la santé au travail et la santé publique. Santé publique, 20(HS), 19-28.

[28] Clot, Y. (2011). Travail et pouvoir d'agir. Paris : PUF

[24] Clot, Y. (2010). Le travail à cœur. Pour en finir avec les risques psychosociaux, Paris : La découverte.

[27] Coutarel, F. (2004). La prévention des troubles musculo-squelettiques en conception: quelles marges de manœuvre pour le déploiement de l'activité ? (Thèse de doctorat, Université Victor Segalen Bordeaux II, Bordeaux, France).

[22] Czarniawska, B. (2009). A Theory of Organazing. Cheltenham: Edward Elgar Publishing.

[8] Estryn-Béhar, M., \& Fouillot, J.P. (1990). Etude de la charge physique du personnel soignant. Analyse du travail des infirmières et aides-soignantes dans 10 services de soins. Documents pour le Médecin du Travail.

[3] Estryn-Behar, M. (2004). Santé, satisfaction au travail et abandon du métier de soignant. Etude PRESST-NEXT. Partie 1.

[11] Estryn-Behar, M., Chaumon, E., Garcia, F., Milanini-Magny, G., Bitot, T., Ravache, AE., \& Deslandes, H. (2011). Isolement, parcellisation du travail et qualité des soins en gériatrie. Activités, 8(1), 77, 103.

[21] Fassier, J.B., \& Durand, M.J. (2010). L'analyse d'implantation des interventions en santé et sécurité au travail. Archives des Maladies Professionnelles et de l'Environnement, 71, 102-107.

[16] Faverge, J-M., Olivier, M., Delahaut J., Stephaneck, P., \& Falmagne J.C. (1970). L'ergonomie des processus industriels. Études de psychologie sociale et industrielle. Université Libre de Bruxelles.

[15] Gonzalez, R, \& Weill-Fassina, A. (2005). Modalités de régulation du processus de travail dans les activités de service en crèche. @ ctivités.

[9] Grosjean, M., \& Lacoste, M. (1999). Communications et intelligence collective. Le travail à l'hôpital. Paris : PUF. 
[18] Guide Aract Ile-de-France (2010). Conditions de travail et prévention des troubles musculo- squelettiques dans les maisons de retraites -. Constats et préconisations. Tiré de l'URL : http://www.aractidf.org/sites/default/files/centredocumentaire/conditions-detravail-prevention-tms-maisons-retraite-aract-idf-oeth.pdf.

[2] Krynen, B., Yeni, I., \& Fournales, R. (2011). Evaluation du dispositif de reclassement des fonctionnaires déclarés inaptes à l'exercice de leurs fonctions pour des raisons de santé (Rapport N¹1-083-01). Inspection générale des affaires sociales et administratives.

[7] Lamy, S., De Gaudemaris, R., Lepage, B., Sobaszek, A., Caroly, S., Kelly-Irving, M., \& Lang, T. (2013). The organizational work factors' effect on mental health among hospital workers is mediated by perceived effort-reward imbalance: Result of a longitudinal study. Journal of Occupational \& Environmental Medicine, 55(7), 809-816.

[10] Martin, C., \& Gadbois, C. (2004). L'ergonomie à l'hôpital. Dans P. Falzon (Edit.), Ergonomie. Paris: Presses Universitaires de France.

[4] Maumet, S., De Gaudemaris, R., Caroly, S., \& Balducci, F. (2005). Facteurs associés à la prévalence des troubles musculo-squelettiques en milieu hospitalier. Recherche des éléments à prendre en compte pour l'évaluation des risques. Archive des maladies professionnelles, 66, 236-243.

[13] Meuleman, F. (2011). Vaincre l'absentéisme. Paris, France : Dunod.

[1] Michel, E., Amar, A., Josselin, V., Caroly, S., Merceron, G., \& De Gaudemaris, R. (2007). Evaluation d'une stratégie pluridisciplinaire de maintien et retour au travail du CHU de Grenoble. Archives des maladies professionnelles et de l'environnement, 68(5), 474481.

[23] Petit, J., \& Dugué, B. (2011). La qualité du travail : un enjeu majeur. Communication présentée lors du 3éme congrès francophone sur les TMS, Grenoble.

[5] Roquelaure, Y., Moisan, S., Brinon, C., \& Juret, I. (2006). Troubles musculo-squelettiques du membre supérieur et des lombalgies dans le secteur santé: données du réseau de surveillance épidémiologique des pays de la Loire de 2002 à 2004 (Rapport), France :LEEST.

[25] Sznelwar L. I., Mascia F. L. \& Bouyer G. (2006). L'empêchement au travail : une source majeure de TMS ? Activités 


\section{Tableaux de l'article}

Tableau 1. Caractéristiques de l'échantillon

\begin{tabular}{|l|c|c|c|c|c|c|}
\hline Hôpitaux & \multicolumn{3}{|c|}{ AS } & \multicolumn{3}{c|}{ IDE } \\
\hline Variables sexe & âges & ancienneté & sexe & âges & ancienneté \\
\hline Service 1 & F & 36 & 6 ans & F & 35 & 13 ans \\
\hline Service 2 & F & 25 & 8 ans & F & 35 & 8 ans \\
\hline Service 3 & F & 37 & 15 ans & F & 24 & $<1$ an \\
\hline
\end{tabular}

Tableau 2. Contextes organisationnels des services de soins observés

\begin{tabular}{|c|c|c|}
\hline \multirow{3}{*}{ 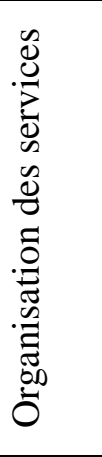 } & $\begin{array}{l}\text { Service } \\
1\end{array}$ & $\begin{array}{l}\text { - Organisation par aile } \\
\text { - Service isolé du reste de l'hôpital } \\
\text { - Service sur deux étages avec la même organisation (même nombre } \\
\text { d'agents et de soignants) }\end{array}$ \\
\hline & $\begin{array}{l}\text { Service } \\
2\end{array}$ & Organisation par couloir sur un étage \\
\hline & $\begin{array}{l}\text { Service } \\
3\end{array}$ & $\begin{array}{l}\text { - } 2 \text { unités fonctionnelles réparties par aile } \\
\text { - Organisation par secteur } \\
\text { - Période de restructuration (ouverture d'une nouvelle aile) } \\
\text { - Service isolé du reste de l'hôpital }\end{array}$ \\
\hline \multirow{3}{*}{ 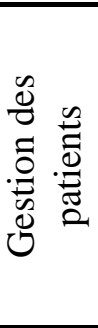 } & $\begin{array}{l}\text { Service } \\
1\end{array}$ & $\begin{array}{l}\text { - Nombre de patients : } 35 \text { par étage } \\
\text { - Patients lourds, dépendants, poly pathologies }\end{array}$ \\
\hline & $\begin{array}{l}\text { Service } \\
2\end{array}$ & $\begin{array}{l}\text { - Nombre de patients : } 20 \\
\text { - Patients provenant très souvent des urgences, état variable, âgée de }+ \\
\text { de } 75 \text { ans }\end{array}$ \\
\hline & $\begin{array}{l}\text { Service } \\
3\end{array}$ & $\begin{array}{l}\text { - Nombre de patients : } 40 \\
\text { - Patients lourds et dépendants }\end{array}$ \\
\hline \multirow{3}{*}{ 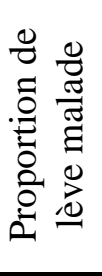 } & $\begin{array}{l}\text { Service } \\
1\end{array}$ & 1 lève malade pour 35 patients \\
\hline & $\begin{array}{l}\text { Service } \\
2\end{array}$ & 1 lève malade pour 20 patients \\
\hline & $\begin{array}{l}\text { Service } \\
3\end{array}$ & $\begin{array}{l}2 \text { lèves malades pour } 40 \text { patients mais le poids maximal autorisé ne } \\
\text { correspond pas aux types de patients }\end{array}$ \\
\hline \multirow{3}{*}{ 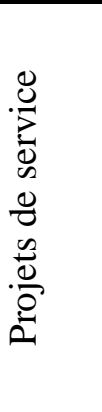 } & $\begin{array}{l}\text { Service } \\
1\end{array}$ & $\begin{array}{l}\text { - Plusieurs projets de service mis en place } \\
\text { - Plusieurs temps de communication dans l'année }\end{array}$ \\
\hline & $\begin{array}{l}\text { Service } \\
2\end{array}$ & $\begin{array}{l}\text { - Plusieurs projets en cours, équipe très dynamique poussée par le } \\
\text { médecin } \\
\text { - Plusieurs temps de communication mais volonté des soignants d'en } \\
\text { avoir plus : demande d'analyse de la pratique avec un psychologue }\end{array}$ \\
\hline & $\begin{array}{l}\text { Service } \\
3\end{array}$ & $\begin{array}{l}\text { - Peu de projet de service, période de restructuration et d'absentéisme } \\
\text { important } \\
\text { - Pas de réunion d'information }\end{array}$ \\
\hline
\end{tabular}

\footnotetext{
${ }^{1}$ Une aile désigne un côté du service : aile sud, aile nord.
} 


\begin{tabular}{|c|c|c|}
\hline \multirow{3}{*}{ 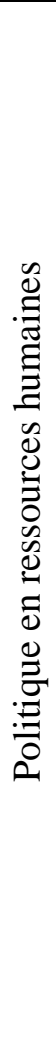 } & $\begin{array}{l}\text { Service } \\
1\end{array}$ & $\begin{array}{l}\text { - Absence de glissement de tâche des ASH } \\
\text { - Travail en } 10 \text { heures } \\
\text { - Travail en binôme si besoin (AS et IDE) mais toilettes patients } \\
\text { réalisées seuls } \\
\text { - Nombre ETP prévu dans le service : } 16 \text { IDE et } 18 \text { pour les AS } \\
\text { - Absentéisme fréquent devenu «la norme » (soit il manque pour les } \\
\text { IDE 2,76 ETP }{ }^{2} \text { et } 0,26 \text { ETP pour les AS) mais possibilité de } \\
\text { remplacement avec le second étage }\end{array}$ \\
\hline & $\begin{array}{l}\text { Service } \\
2\end{array}$ & $\begin{array}{l}\text { - Glissement de tâches des ASH } \\
\text { - Travail en } 10 \text { heures } \\
\text { - Travail en collectif entre corps de métier : AS font les toilettes à } 2 \\
\text { - Nombre ETP prévu dans le service : } 9,60 \text { IDE et } 9,5 \text { pour les AS } \\
\text { - Période d'absentéisme (soit il manque pour les IDE } 0,48 \text { ETP et } 0,47 \\
\text { ETP pour les AS) mais service qui fonctionne bien avec possibilité de } \\
\text { remplacement }\end{array}$ \\
\hline & $\begin{array}{l}\text { Service } \\
3\end{array}$ & $\begin{array}{l}\text { - Absence de glissement de tâche des ASH } \\
\text { - Travail en } 10 \text { heures } \\
\text { - Pas de travail collectif (nombreux nouveaux IDE) } \\
\text { - Conflits de génération entre les soignants } \\
\text { - Nombre ETP prévu dans le service : } 17,5 \text { IDE et 18,5 pour les AS } \\
\text { - Absentéisme récurrent (soit il manque pour les IDE } 0,15 \text { ETP et } 0,46 \\
\text { ETP pour les AS). Soignants peu remplacés par le pole, ce qui implique } \\
\text { de nombreux changements de planning }\end{array}$ \\
\hline
\end{tabular}

Tableau 3. Catégorisation des échanges des soignants observés afin de se répartir la charge de travail

\begin{tabular}{|l|l|l|l|l|l|l|l|}
\hline & \multicolumn{2}{|l|}{ Service 1 } & \multicolumn{2}{l|}{ Service 2 } & \multicolumn{2}{l|}{ Service 3 } & \\
\hline Catégories & AS & IDE & AS & IDE & AS & IDE & Somme \\
\hline Aide & 8 & 10 & 8 & 14 & 8 & 14 & $\mathbf{6 2}$ \\
\hline Matériel & 4 & 7 & 2 & 7 & 4 & 3 & $\mathbf{2 7}$ \\
\hline $\begin{array}{l}\text { Organisation } \\
\text { des soins }\end{array}$ & 10 & 8 & 9 & 14 & 3 & 9 & $\mathbf{5 3}$ \\
\hline $\begin{array}{l}\text { Coordination } \\
\text { des soins }\end{array}$ & 19 & 20 & 5 & 17 & 34 & 18 & $\mathbf{1 1 3}$ \\
\hline
\end{tabular}

\footnotetext{
${ }^{2}$ Soit le nombre Equivalent Temps Plein (ETP) par lits.
} 